\title{
THE ORLICZ SPACE OF ENTIRE SEQUENCES
}

\section{K. CHANDRASEKHARA RAO and N. SUBRAMANIAN}

Received 24 November 2003

Let $\Gamma$ denote the space of all entire sequences and $\wedge$ the space of all analytic sequences. This paper is devoted to the study of the general properties of Orlicz space $\Gamma_{M}$ of $\Gamma$.

2000 Mathematics Subject Classification: 46A45.

1. Introduction. An Orlicz function is a function $M:[0, \infty) \rightarrow[0, \infty)$ which is continuous, nondecreasing, and convex with $M(0)=0, M(x)>0$ for $x>0$, and $M(x) \rightarrow \infty$ as $x \rightarrow \infty$. If the convexity of Orlicz function $M$ is replaced by $M(x+y) \leq M(x)+M(y)$, then this function is called a modulus function, defined and discussed by Ruckle [5] and Maddox [4].

An Orlicz function $M$ is said to satisfy the $\Delta_{2}$-condition for all values of $u$ if there exists a constant $K>0$ such that $M(2 u) \leq K M(u)(u \geq 0)$. The $\Delta_{2}$-condition is equivalent to $M(\ell u) \leq K . \ell M(u)$, for all values of $u$ and for $\ell>1$.

An Orlicz function $M$ can always be represented in the following integral form: $M(x)=\int_{0}^{x} q(t) d t$, where $q$, known as the kernel of $M$, is right-differentiable for $t \geq 0$, $q(0)=0, q(t)>0$ for $t>0, q$ is nondecreasing, and $q(t) \rightarrow \infty$ as $t \rightarrow \infty$. Lindenstrauss and Tzafriri [3] used the idea of Orlicz function to construct the Orlicz sequence space

$$
\ell_{M}=\left\{x \in w: \sum_{k=1}^{\infty} M\left(\frac{\left|x_{k}\right|}{\rho}\right)<\infty \text {, for some } \rho>0\right\},
$$

where $w=\{$ all complex sequences $\}$.

The space $\ell_{M}$ with the norm

$$
\|x\|=\inf \left\{\rho>0: \sum_{k=1}^{\infty} M\left(\frac{\left|x_{k}\right|}{\rho}\right) \leq 1\right\}
$$

becomes a Banach space which is called an Orlicz sequence space.

2. A complex sequence whose $k$ th term is $x_{k}$ will be denoted by $\left(x_{k}\right)$ or $x$. A sequence $x=\left(x_{k}\right)$ is said to be analytic if $\sup _{(k)}\left|x_{k}\right|^{1 / k}<\infty$. The vector space of all analytic sequences will be denoted by $\wedge$. A sequence $x$ is called an entire sequence if $\lim _{k \rightarrow \infty}\left|x_{k}\right|^{1 / k}=0$. The vector space of all entire sequences will be denoted by $\Gamma$.

DEFINITION 2.1. The space consisting of all sequences $x$ in $w$ such that $M\left(\left|x_{k}\right|^{1 / k} /\right.$ $\rho) \rightarrow 0$ as $k \rightarrow \infty$ for some arbitrarily fixed $\rho>0$ is denoted by $\Gamma_{M}$, with $M$ being a modulus function. In other words, $\left\{M\left(\left|x_{k}\right|^{1 / k} / \rho\right)\right\}$ is a null sequence. The space $\Gamma_{M}$ is 
a metric space with the metric

$$
d(x, y)=\sup _{(k)} M\left(\frac{\left|x_{k}-y_{k}\right|^{1 / k}}{\rho}\right)
$$

for all $x=\left\{x_{k}\right\}$ and $y=\left\{y_{k}\right\}$ in $\Gamma_{M}$.

Given a sequence $x=\left\{x_{k}\right\}$ whose $n$th section is the sequence $x^{(n)}=\left\{x_{1}, x_{2}, \ldots, x_{n}, 0\right.$, $0, \ldots\}, \delta^{(n)}=(0,0, \ldots, 1,0,0, \ldots)$, with 1 in the $n$th place and zeros elsewhere; let $\Phi=$ \{all finite sequences\}.

An FK-space (or a metric space) $X$ is said to have AK property if $\left(\delta^{(n)}\right)$ is a Schauder basis for $X$. Or equivalently $x^{(n)} \rightarrow x$.

The space is said to have or be an AD space if $\Phi$ is dense in $X$.

We note that $\mathrm{AK}$ implies $\mathrm{AD}$ by [1].

If $X$ is a sequence space, we give the following definitions:

(i) $X^{\prime}=$ the continuous dual of $X$;

(ii) $X^{\alpha}=\left\{a=\left(a_{k}\right): \sum_{k=1}^{\infty}\left|a_{k} x_{k}\right|<\infty\right.$, for each $\left.x \in X\right\}$;

(iii) $X^{\beta}=\left\{a=\left(a_{k}\right): \sum_{k=1}^{\infty} a_{k} x_{k}\right.$ is convergent, for each $\left.x \in X\right\}$;

(iv) $X^{\gamma}=\left\{a=\left(a_{k}\right): \sup _{(n)}\left|\sum_{k=1}^{n} a_{k} x_{k}\right|<\infty\right.$, for each $\left.x \in X\right\}$;

(v) let $X$ be an FK-space $\supset \Phi$, then $X^{f}=\left\{f\left(\delta^{(n)}\right): f \in X^{\prime}\right\} . X^{\alpha}, X^{\beta}$, and $X^{\gamma}$ are called the $\alpha$ - (or Köthe-Toeplitz-) dual of $X, \beta$ - (or generalized-Köthe-Toeplitz-) dual of $X$, and $\gamma$-dual of $X$, respectively.

Note that $X^{\alpha} \subset X^{\beta} \subset X^{\gamma}$. If $X \subset Y$, then $Y^{\mu} \subset X^{\mu}$, for $\mu=\alpha$, $\beta$, or $\gamma$.

LEMMA 2.2 (see [6, Theorem 7.2.7]). Let $X$ be an FK-space $\supset \Phi$. Then

(i) $X^{\gamma} \subset X^{f}$;

(ii) if $X$ has $A K, X^{\beta}=X^{f}$;

(iii) if $X$ has $A D, X^{\beta}=X^{\gamma}$.

We note that $\Gamma^{\alpha}=\Gamma^{\beta}=\Gamma^{\gamma}=\wedge$.

Proposition 2.3. $\Gamma \subset \Gamma_{M}$, with the hypothesis that $M\left(\left|x_{k}\right|^{1 / k} / \rho\right) \leq\left|x_{k}\right|^{1 / k}$.

Proof. Let $x \in \Gamma$. Then we have the following implications:

$$
\left|x_{k}\right|^{1 / k} \longrightarrow 0 \quad \text { as } k \rightarrow \infty
$$

But $M\left(\left|x_{k}\right|^{1 / k} / \rho\right) \leq\left|x_{k}\right|^{1 / k}$, by our assumption, implies that

$$
\begin{aligned}
& M\left(\frac{\left|x_{k}\right|^{1 / k}}{\rho}\right) \longrightarrow 0 \quad \text { as } k \longrightarrow \infty(\text { by }(2.2)) \\
& \quad \Longrightarrow x \in \Gamma_{M} \\
& \quad \Longrightarrow \Gamma \subset \Gamma_{M} .
\end{aligned}
$$

This completes the proof. 
Proposition 2.4. $\Gamma_{M}$ has $A K$ where $M$ is a modulus function.

Proof. Let $x=\left\{x_{k}\right\} \in \Gamma_{M}$, but then $\left\{M\left(\left|x_{k}\right|^{1 / k} / \rho\right)\right\} \in \Gamma$, and hence

$$
\sup _{k \geq n+1} M\left(\frac{\left|x_{k}\right|^{1 / k}}{\rho}\right) \longrightarrow 0 \quad \text { as } n \longrightarrow \infty .
$$

By using (2.4), $d\left(x, x^{[n]}\right)=\sup _{k \geq n+1} M\left(\left|x_{k}\right|^{1 / k} / \rho\right) \rightarrow 0$ as $n \rightarrow \infty$, which implies that $x^{[n]} \rightarrow x$ as $n \rightarrow \infty$, implying that $\Gamma_{M}$ has AK. This completes the proof.

Proposition 2.5. $\Gamma_{M}$ is solid.

Proof. Let $\left|x_{k}\right| \leq\left|y_{k}\right|$ and let $y=\left(y_{k}\right) \in \Gamma_{M} . M\left(\left|x_{k}\right|^{1 / k} / \rho\right) \leq M\left(\left|y_{k}\right|^{1 / k} / \rho\right)$, because $M$ is nondecreasing. But $M\left(\left|y_{k}\right|^{1 / k} / \rho\right) \in \Gamma$ because $y \in \Gamma_{M}$. That is, $M\left(\left|y_{k}\right|^{1 / k} / \rho\right) \rightarrow 0$ as $k \rightarrow \infty$ and $M\left(\left|x_{k}\right|^{1 / k} / \rho\right) \rightarrow 0$ as $k \rightarrow \infty$. Therefore $x=\left\{x_{k}\right\} \in \Gamma_{M}$. This completes the proof.

Proposition 2.6. Let $M$ be an Orlicz function which satisfies the $\Delta_{2}$-condition. Then $\Gamma \subset \Gamma_{M}$.

Proof. Let

$$
x \in \Gamma .
$$

Then $\left|x_{k}\right|^{1 / k} \leq \varepsilon$ for sufficiently large $k$ and every $\varepsilon>0$. But then by taking $\rho \geq 1 / 2$,

$$
\begin{aligned}
M\left(\frac{\left|x_{k}\right|^{1 / k}}{\rho}\right) & \leq M\left(\frac{\varepsilon}{\rho}\right) \quad \text { (because } M \text { is nondecreasing) } \\
\leq M(2 \varepsilon) & \\
\Rightarrow M\left(\frac{\left|x_{k}\right|^{1 / k}}{\rho}\right) & \leq K M(\varepsilon) \quad\left(\text { by the } \Delta_{2} \text {-condition, for some } K>0\right) \\
& \leq \varepsilon \quad\left(\text { by defining } M(\varepsilon)<\frac{\varepsilon}{k}\right) \\
\Longrightarrow M\left(\frac{\left|x_{k}\right|^{1 / k}}{\rho}\right) & \longrightarrow 0 \quad \text { as } k \rightarrow \infty .
\end{aligned}
$$

Hence $x \in \Gamma_{M}$.

From (2.5) and since

$$
x \in \Gamma_{M},
$$

we get

$$
\Gamma \subset \Gamma_{M}
$$

This completes the proof.

Proposition 2.7. If $M$ is a modulus function, then $\Gamma_{M}$ is a linear set over the set of complex numbers $\mathbb{C}$. 
Proof. Let $x, y \in \Gamma_{M}$ and $\alpha, \beta \in \mathbb{C}$. In order to prove the result, we need to find some $\rho_{3}$ such that

$$
M\left(\frac{\left|\alpha x_{k}+\beta y_{k}\right|^{1 / k}}{\rho_{3}}\right) \rightarrow 0 \quad \text { as } k \rightarrow \infty
$$

Since $x, y \in \Gamma_{M}$, there exist some positive $\rho_{1}$ and $\rho_{2}$ such that

$$
\begin{aligned}
& M\left(\frac{\left|x_{k}\right|^{1 / k}}{\rho_{1}}\right) \longrightarrow 0 \text { as } k \longrightarrow \infty \\
& M\left(\frac{\left|y_{k}\right|^{1 / k}}{\rho_{2}}\right) \longrightarrow 0 \text { as } k \rightarrow \infty
\end{aligned}
$$

Since $M$ is a nondecreasing modulus function, we have

$$
\begin{aligned}
M\left(\frac{\left|\alpha x_{k}+\beta y_{k}\right|^{1 / k}}{\rho_{3}}\right) & \leq M\left(\frac{\left|\alpha x_{k}\right|^{1 / k}}{\rho_{3}}+\frac{\left|\beta y_{k}\right|^{1 / k}}{\rho_{3}}\right) \\
& \leq M\left(\frac{|\alpha|^{1 / k}\left|x_{k}\right|^{1 / k}}{\rho_{3}}+\frac{|\beta|^{1 / k}\left|y_{k}\right|^{1 / k}}{\rho_{3}}\right) \\
& \leq M\left(\frac{|\alpha|\left|x_{k}\right|^{1 / k}}{\rho_{3}}+\frac{|\beta|\left|y_{k}\right|^{1 / k}}{\rho_{3}}\right)
\end{aligned}
$$

Take $\rho_{3}$ such that

$$
\frac{1}{\rho_{3}}=\min \left\{\frac{1}{|\alpha|} \frac{1}{\rho_{1}}, \frac{1}{|\beta|} \frac{1}{\rho_{2}}\right\}
$$

Then

$$
\begin{aligned}
M\left(\frac{\left|\alpha x_{k}+\beta y_{k}\right|^{1 / k}}{\rho_{3}}\right) & \leq M\left(\frac{\left|x_{k}\right|^{1 / k}}{\rho_{1}}+\frac{\left|y_{k}\right|^{1 / k}}{\rho_{2}}\right) \\
& \leq M\left(\frac{\left|x_{k}\right|^{1 / k}}{\rho_{1}}\right)+M\left(\frac{\left|y_{k}\right|^{1 / k}}{\rho_{2}}\right) \\
& \longrightarrow 0(\text { by (2.10)) }
\end{aligned}
$$

Hence

$$
M\left(\frac{\left|\alpha x_{k}+\beta y_{k}\right|^{1 / k}}{\rho_{3}}\right) \rightarrow 0 \quad \text { as } k \longrightarrow \infty
$$

So $(\alpha x+\beta y) \in \Gamma_{M}$. Therefore $\Gamma_{M}$ is linear. This completes the proof. 
DEFINITION 2.8. Let $p=\left(p_{k}\right)$ be any sequence of positive real numbers. Then

$$
\Gamma_{M}(p)=\left\{x=\left\{x_{k}\right\}:\left(M\left(\frac{\left|x_{k}\right|^{1 / k}}{\rho}\right)\right)^{p_{k}} \longrightarrow 0 \text { as } k \longrightarrow \infty\right\} .
$$

Suppose that $p_{k}$ is a constant for all $k$, then $\Gamma_{M}(p)=\Gamma_{M}$.

Proposition 2.9. Let $0 \leq p_{k} \leq q_{k}$ and let $\left\{q_{k} / p_{k}\right\}$ be bounded. Then $\Gamma_{M}(q) \subset \Gamma_{M}(p)$.

Proof. Let

$$
\begin{gathered}
x \in \Gamma_{M}(q), \\
\left(M\left(\frac{\left|x_{k}\right|^{1 / k}}{\rho}\right)\right)^{q_{k}} \longrightarrow 0 \text { as } k \longrightarrow \infty .
\end{gathered}
$$

Let $t_{k}=\left(M\left(\left|x_{k}\right|^{1 / k} / \rho\right)\right)^{q_{k}}$ and $\lambda_{k}=p_{k} / q_{k}$. Since $p_{k} \leq q_{k}$, we have $0 \leq \lambda_{k} \leq 1$.

Take $0<\lambda<\lambda_{k}$. Define

$$
\begin{gathered}
u_{k}= \begin{cases}t_{k} & \left(t_{k} \geq 1\right) \\
0 & \left(t_{k}<1\right),\end{cases} \\
v_{k}= \begin{cases}0 & \left(t_{k} \geq 1\right) \\
t_{k} & \left(t_{k}<1\right),\end{cases} \\
t_{k}=u_{k}+v_{k}, \quad t_{k}^{\lambda_{k}}=u_{k}^{\lambda_{k}}+v_{k}^{\lambda_{k}} .
\end{gathered}
$$

Now it follows that

$$
u_{k}^{\lambda_{k}} \leq u_{k} \leq t_{k}, \quad v_{k}^{\lambda_{k}} \leq v_{k}^{\lambda}
$$

Since $t_{k}^{\lambda_{k}}=u_{k}^{\lambda_{k}}+v_{k}^{\lambda_{k}}$, then $t_{k}^{\lambda_{k}} \leq t_{k}+v_{k}^{\lambda}$.

$$
\begin{gathered}
\left(M\left(\frac{\left|x_{k}\right|^{1 / k}}{\rho}\right)^{q_{k}}\right)^{\lambda_{k}} \leq\left(M\left(\frac{\left|x_{k}\right|^{1 / k}}{\rho}\right)\right)^{q_{k}} \\
\Rightarrow\left(M\left(\frac{\left|x_{k}\right|^{1 / k}}{\rho}\right)^{q_{k}}\right)^{p_{k} / q_{k}} \leq\left(M\left(\frac{\left|x_{k}\right|^{1 / k}}{\rho}\right)\right)^{q_{k}} \\
\Rightarrow\left(M\left(\frac{\left|x_{k}\right|^{1 / k}}{\rho}\right)\right)^{p_{k}} \leq\left(M\left(\frac{\left|x_{k}\right|^{1 / k}}{\rho}\right)\right)^{q_{k}} .
\end{gathered}
$$

But

$$
\left(M\left(\frac{\left|x_{k}\right|^{1 / k}}{\rho}\right)\right)^{q_{k}} \longrightarrow 0 \quad(\text { by }(2.17))
$$


Hence $\left(M\left(\left|x_{k}\right|^{1 / k} / \rho\right)\right)^{p_{k}} \rightarrow 0$ as $k \rightarrow \infty$. Hence

$$
x \in \Gamma_{M}(p) .
$$

From (2.16) and (2.22), we get

$$
\Gamma_{M}(q) \subset \Gamma_{M}(p)
$$

This completes the proof.

Proposition 2.10. (a) Let $0<\inf p_{k} \leq p_{k} \leq 1$. Then $\Gamma_{M}(p) \subset \Gamma_{M}$.

(b) Let $1 \leq p_{k} \leq \sup p_{k}<\infty$. Then $\Gamma_{M} \subset \Gamma_{M}(p)$.

Proof. (a) Let $x \in \Gamma_{M}(p)$,

$$
\left(M\left(\frac{\left|x_{k}\right|^{1 / k}}{\rho}\right)\right)^{p_{k}} \longrightarrow 0 \text { as } k \longrightarrow \infty .
$$

Since $0<\inf p_{k} \leq p_{k} \leq 1$,

$$
\left(M\left(\frac{\left|x_{k}\right|^{1 / k}}{\rho}\right)\right) \leq\left(M\left(\frac{\left|x_{k}\right|^{1 / k}}{\rho}\right)\right)^{p_{k}},
$$

From (2.24) and (2.25) it follows that

$$
x \in \Gamma_{M} .
$$

Thus

$$
\Gamma_{M}(p) \subset \Gamma_{M}
$$

We have thus proven (a).

(b) Let $p_{k} \geq 1$ for each $k$ and $\sup p_{k}<\infty$ and let $x \in \Gamma_{M}$.

$$
M\left(\frac{\left|x_{k}\right|^{1 / k}}{\rho}\right) \rightarrow 0 \text { as } k \rightarrow \infty
$$

Since $1 \leq p_{k} \leq \sup p_{k}<\infty$, we have

$$
\begin{gathered}
\left(M\left(\frac{\left|x_{k}\right|^{1 / k}}{\rho}\right)\right)^{p_{k}} \leq\left(M\left(\frac{\left|x_{k}\right|^{1 / k}}{\rho}\right)\right) \\
\left(M\left(\frac{\left|x_{k}\right|^{1 / k}}{\rho}\right)\right)^{p_{k}} \longrightarrow 0 \text { as } k \rightarrow \infty \text { (by using (2.28)). }
\end{gathered}
$$

Therefore $x \in \Gamma_{M}(p)$. This completes the proof. 
Proposition 2.11. Let $0<p_{k} \leq q_{k}<\infty$ for each $k$. Then $\Gamma_{M}(p) \subseteq \Gamma_{M}(q)$.

Proof. Let $x \in \Gamma_{M}(p)$

$$
\left(M\left(\frac{\left|x_{k}\right|^{1 / k}}{\rho}\right)\right)^{p_{k}} \longrightarrow 0 \text { as } k \longrightarrow \infty
$$

This implies that $\left(M\left(\left|x_{k}\right|^{1 / k} / \rho\right)\right) \leq 1$ for sufficiently large $k$. Since $M$ is nondecreasing, we get

$$
\begin{aligned}
& \left(M\left(\frac{\left|x_{k}\right|^{1 / k}}{\rho}\right)\right)^{q_{k}} \leq\left(M\left(\frac{\left|x_{k}\right|^{1 / k}}{\rho}\right)\right)^{p_{k}} \\
& \quad \Longrightarrow\left(M\left(\frac{\left|x_{k}\right|^{1 / k}}{\rho}\right)\right)^{q_{k}} \longrightarrow 0 \text { as } k \longrightarrow \infty \text { (by using (2.30)). }
\end{aligned}
$$

Since $x \in \Gamma_{M}(q)$, hence $\Gamma_{M}(p) \subseteq \Gamma_{M}(q)$. This completes the proof.

Proposition 2.12. $\Gamma_{M}(p)$ is $r$-convex for all $r$, where $0 \leq r \leq \inf p_{k}$. Moreover, if $p_{k}=p \leq 1$ for all $k$, then they are $p$-convex.

Proof. We will prove the theorem for $\Gamma_{M}(p)$. Let $x \in \Gamma_{M}(p)$ and $r \in\left(0, \lim _{n \rightarrow \infty} \inf p_{n}\right)$. Then, there exists $k_{0}$ such that $r \leq p_{k}$ for all $k>k_{0}$.

Now, define

$$
g^{*}(x)=\inf \left\{\rho: M\left(\frac{\left|x_{k}-y_{k}\right|^{1 / k}}{\rho}\right)^{r}+M\left(\frac{\left|x_{k}-y_{k}\right|^{1 / k}}{\rho}\right)^{p_{n}}\right\}
$$

Since $r \leq p_{k} \leq 1$ for all $k>k_{0}, g^{*}$ is subadditive. Further, for $0 \leq|\lambda| \leq 1$,

$$
|\lambda|^{p_{k}} \leq|\lambda|^{r} \quad \forall k>k_{0} .
$$

Therefore, for each $\lambda$, we have

$$
g^{*}(\lambda x) \leq|\lambda|^{r} \cdot g^{*}(x)
$$

Now, for $0<\delta<1$,

$$
U=\left\{x: g^{*}(x) \leq \delta\right\},
$$

which is an absolutely $r$-convex set, for

$$
|\lambda|^{r}+|\mu|^{r} \leq 1, \quad x, y \in U .
$$


Now

$$
\begin{aligned}
g^{*}(\lambda x+\mu y) & \leq g^{*}(\lambda x)+g^{*}(\mu y) \\
& \leq|\lambda|^{r} g^{*}(x)+|\mu|^{r} g^{*}(y) \\
& \leq|\lambda|^{r} \delta+|\mu|^{r} \delta \quad(\text { using (2.34) and (2.35)) } \\
& \leq\left(|\lambda|^{r}+|\mu|^{r}\right) \delta \\
& \leq 1 \cdot \delta \quad(\text { by using (2.36)) } \\
& \leq \delta
\end{aligned}
$$

If $p_{k}=p \leq 1$ for all $k$, then for $0<r<1, U=\left\{x: g^{*}(x) \leq \delta\right\}$ is an absolutely $p$-convex set. This can be obtained by a similar analysis and therefore we omit the details. This completes the proof.

Proposition 2.13. $\left(\Gamma_{M}\right)^{\beta}=\wedge$.

\section{PROOF}

STEP 1. $\Gamma \subset \Gamma_{M}$ by Proposition 2.3; this implies that $\left(\Gamma_{M}\right)^{\beta} \subset \Gamma^{\beta}=\wedge$. Therefore,

$$
\left(\Gamma_{M}\right)^{\beta} \subset \wedge
$$

STEP 2. Let $y \in \wedge$. Then $\left|y_{k}\right|<M^{k}$ for all $k$ and for some constant $M>0$.

Let $x \in \Gamma_{M}$. Then $M\left(\left|x_{k}\right|^{1 / k} / \rho\right) \rightarrow 0$ as $k \rightarrow \infty$. Hence $M\left(\left|x_{k}\right|^{1 / k} / \rho\right)<\varepsilon$ for given $\varepsilon>0$ for sufficiently large $k$.

Take $\varepsilon=1 / 2 M$ so that $M\left(\left|x_{k}\right| / \rho\right)<1 /(2 M)^{k}$.

But then $M\left(\left|x_{k} y_{k}\right| / \rho\right) \leq 1 / 2^{k}$ so that $\sum_{k=1}^{\infty} M\left(\left|x_{k} y_{k}\right| / \rho\right)$ converges. Therefore $\sum_{k=1}^{\infty} M\left(x_{k} y_{k} / \rho\right)$ converges. Hence $\sum_{k=1}^{\infty} x_{k} y_{k}$ converges so that $y \in\left(\Gamma_{M}\right)^{\beta}$. Thus

$$
\wedge \subset\left(\Gamma_{M}\right)^{\beta} \text {. }
$$

STEP 3. From (2.38) and (2.39), we obtain

$$
\left(\Gamma_{M}\right)^{\beta}=\wedge
$$

This completes the proof.

Proposition 2.14. $\left(\Gamma_{M}\right)^{\mu}=\wedge$ for $\mu=\alpha, \beta, \gamma, f$.

\section{PROOF}

STEP 1. $\Gamma_{M}$ has AK by Proposition 2.4. Hence, by Lemma 2.2(i), we get $\left(\Gamma_{M}\right)^{\beta}=\left(\Gamma_{M}\right)^{f}$. But $\left(\Gamma_{M}\right)^{\beta}=\wedge$. Hence

$$
\left(\Gamma_{M}\right)^{f}=\wedge
$$

STEP 2. Since AK implies AD, hence by Lemma 2.2(iii) we get $\left(\Gamma_{M}\right)^{\beta}=\left(\Gamma_{M}\right)^{\gamma}$. Therefore

$$
\left(\Gamma_{M}\right)^{\gamma}=\wedge .
$$


STEP 3. $\Gamma_{M}$ is normal by Proposition 2.5. Hence, by [2, Proposition 2.7], we get

$$
\left(\Gamma_{M}\right)^{\alpha}=\left(\Gamma_{M}\right)^{\gamma}=\wedge
$$

From (2.41), (2.42), and (2.43), we have

$$
\left(\Gamma_{M}\right)^{\alpha}=\left(\Gamma_{M}\right)^{\beta}=\left(\Gamma_{M}\right)^{\gamma}=\left(\Gamma_{M}\right)^{f}=\wedge .
$$

Proposition 2.15. The dual space of $\Gamma_{M}$ is $\wedge$. In other words, $\Gamma_{M}^{*}=\wedge$.

Proof. We recall that $\delta^{k}$ has 1 in the $k$ th place and zeros elsewhere, with

$$
\begin{aligned}
x=\delta^{k}, \quad\left\{M\left(\frac{\left|x_{k}\right|^{1 / k}}{\rho}\right)\right\} & =\left\{\frac{M(0)^{1}}{\rho}, \frac{M(0)^{1 / 2}}{\rho}, \ldots, \frac{M(1)^{1 / k}}{\rho}, \frac{M(0)^{1 /(k+1)}}{\rho}, \ldots\right\} \\
& =\left\{0,0, \ldots, \frac{M(1)^{1 / k}}{\rho}, 0, \ldots\right\}
\end{aligned}
$$

which is a null sequence. Hence $\delta^{k} \in \Gamma_{M} . f(x)=\sum_{k=1}^{\infty} x_{k} y_{k}$ with $x \in \Gamma_{M}$ and $f \in \Gamma_{M}^{*}$, where $\Gamma_{M}^{*}$ is the dual space of $\Gamma_{M}$. Take $x=\delta^{k} \in \Gamma_{M}$. Then

$$
\left|y_{k}\right| \leq\|f\| d\left(\delta^{k}, 0\right)<\infty \quad \forall k
$$

Thus $\left(y_{k}\right)$ is a bounded sequence and hence an analytic sequence. In other words, $y \in \wedge$. Therefore $\Gamma_{M}^{*}=\wedge$. This completes the proof.

LEMmA 2.16 [6, Theorem 8.6.1]. $Y \supset X \Leftrightarrow Y^{f} \subset X^{f}$, where $X$ is an AD-space and $Y$ an FK-space.

Proposition 2.17. Let $Y$ be any $F K$-space $\supset \Phi$. Then $Y \supset \Gamma_{M}$ if and only if the sequence $\delta^{(k)}$ is weakly analytic.

Proof. The following implications establish the result: since $\Gamma_{M}$ has $\mathrm{AD}$ and by Lemma 2.16,

$$
\begin{aligned}
Y \supset \Gamma_{M} & \Longleftrightarrow Y^{f} \subset\left(\Gamma_{M}\right)^{f} \\
& \Longleftrightarrow Y^{f} \subset \wedge \quad\left(\text { since }\left(\Gamma_{M}\right)^{f}=\wedge\right) \\
& \Longleftrightarrow \text { for each } f \in Y^{\prime}, \text { the topological dual of } Y \cdot f\left(\delta^{(k)}\right) \in \wedge \\
& \Longleftrightarrow f\left(\delta^{(k)}\right) \text { is analytic } \\
& \Longleftrightarrow \delta^{(k)} \text { is weakly analytic, }
\end{aligned}
$$

this completes the proof. 


\section{REFERENCES}

[1] H. I. Brown, The summability field of a perfect l-l method of summation, J. Anal. Math. 20 (1967), 281-287.

[2] P. K. Kamthan and M. Gupta, Sequence Spaces and Series, Lecture Notes in Pure and Applied Mathematics, vol. 65, Marcel Dekker, New York, 1981.

[3] J. Lindenstrauss and L. Tzafriri, On Orlicz sequence spaces, Israel J. Math. 10 (1971), 379390.

[4] I. J. Maddox, Sequence spaces defined by a modulus, Math. Proc. Cambridge Philos. Soc. 100 (1986), no. 1, 161-166.

[5] W. H. Ruckle, FK spaces in which the sequence of coordinate vectors is bounded, Canad. J. Math. 25 (1973), 973-978.

[6] A. Wilansky, Summability through Functional Analysis, North-Holland Mathematics Studies, vol. 85, North-Holland Publishing, Amsterdam, 1984.

K. Chandrasekhara Rao: Srinivasa Ramanujan Centre, Shanmugha Arts, Science, Technology, and Research Academy (SASTRA), Kumbakonam 612 001, India

E-mail address: kcrao2008@yahoo.co.in

N. Subramanian: Deptartment of Mathematics, Shanmugha Arts, Science, Technology, and Research Academy (SASTRA), Thanjavur 613 402, India

E-mail address: nsmaths@yahoo.com 


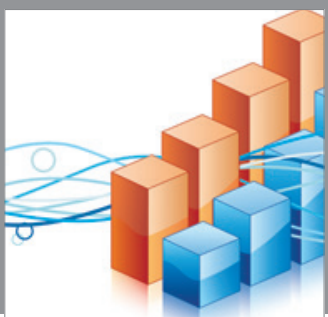

Advances in

Operations Research

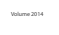

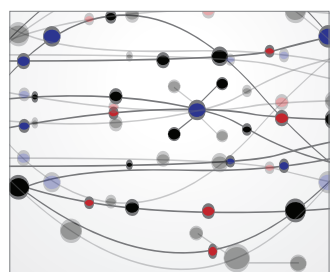

\section{The Scientific} World Journal
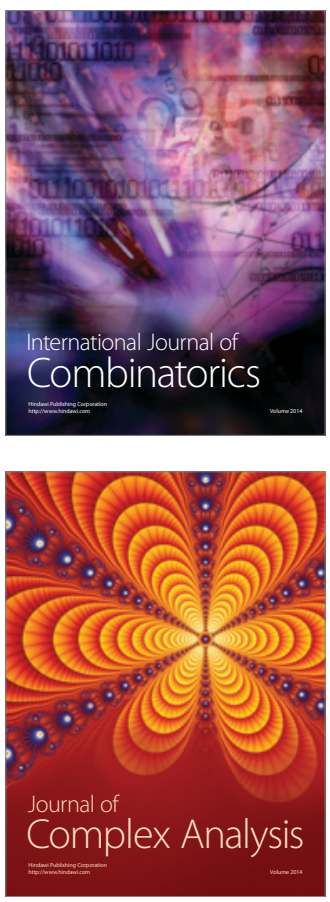

International Journal of

Mathematics and

Mathematical

Sciences
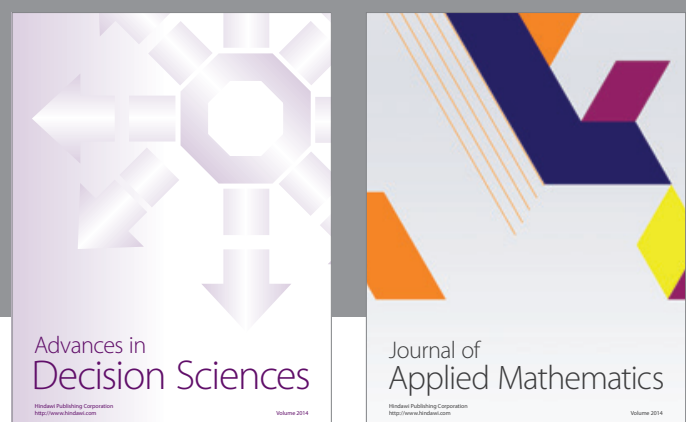

Journal of

Applied Mathematics
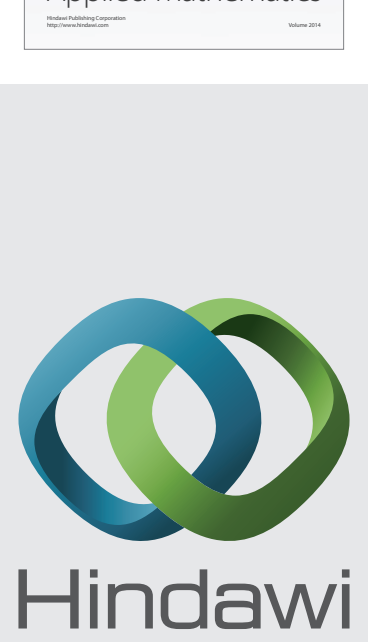

Submit your manuscripts at http://www.hindawi.com
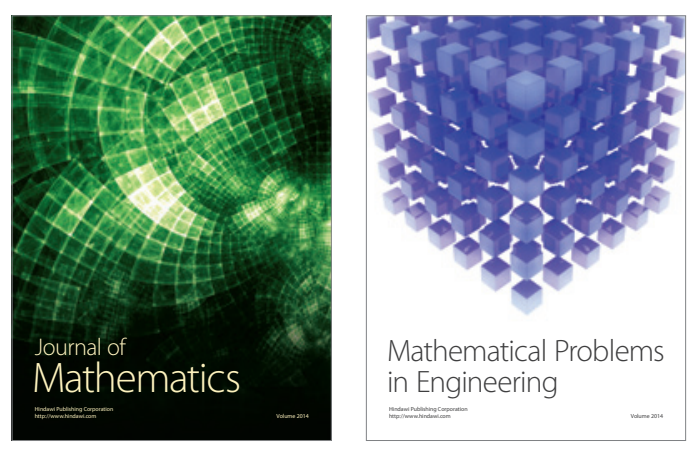

Mathematical Problems in Engineering
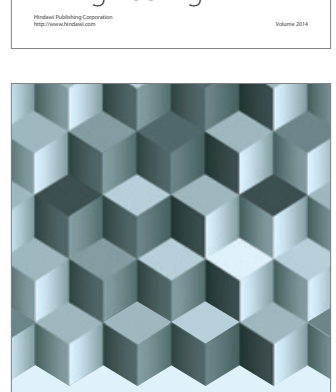

Journal of

Function Spaces
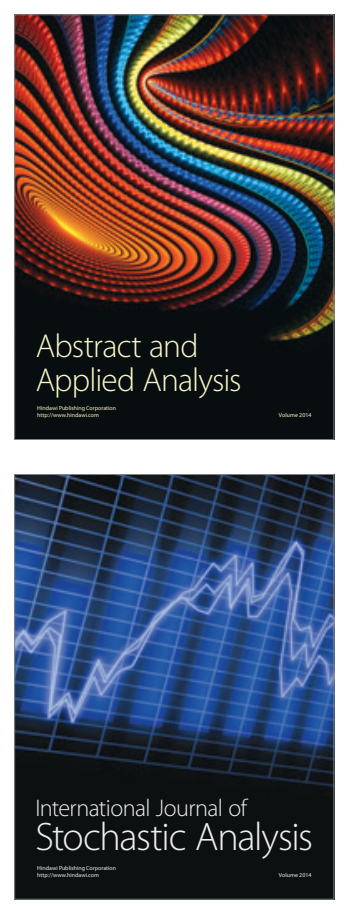

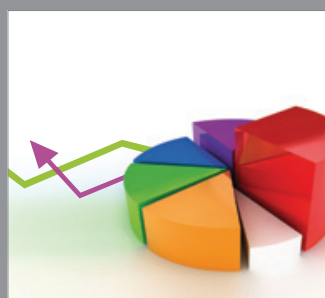

ournal of

Probability and Statistics

Promensencen
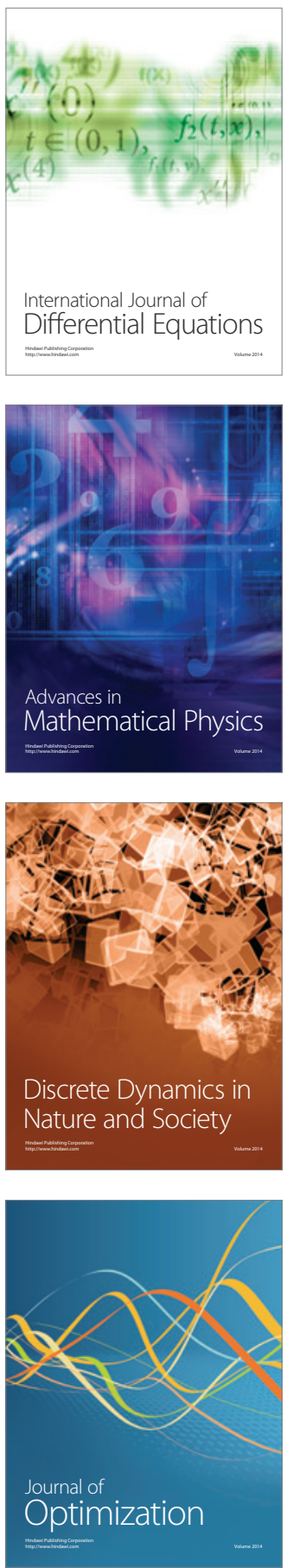